\title{
Polymer Chain Dynamics III. Intermittent Orientational Relaxation of Terminal Segment on Polystyrene Chain
}

\author{
Fumiaki TsunOMORI and Hideharu UsHIKI \\ Graduate School of Bio-Applications and Systems Engineering, Tokyo University of Agriculture and Technology, Fuchu, Tokyo 183, Japan
}

(Received November 16, 1995)

\begin{abstract}
Polystyrene having an anthryl group attached at the end of a chain, whose degree of polymerization is 550, was used. We have found that the most appropriate orientational auto-correlation function for a terminal segment on a chain is hyperbolic function $r(t) \sim r_{0}\left(t / \tau_{\text {eff }}\right)^{-\beta}$. This behavior indicates the primary process of orientational relaxation of chain segments to be the intermittent process. The distribution of the state density of a terminal segment is in agreement with that of "inner" segment according to the reference temperature $T_{\mathrm{r}}$, which reflects to the characteristic activation energy of orientational relaxation of segments. Therefore the characteristic activation energy of orientational relaxation of a segment is independent of position on a chain. The values of minimum reaction radii $\xi_{0}$ of a terminal segment were about twice times larger than that of a central segment. This behavior can be reduced to the degree of freedom of segmental motion on the basis of computer simulation. Therefore $\xi_{0}$ corresponds not only the apparent activation energy of solvent molecules but the degree of freedom of segmental motion on a polymer chain.
\end{abstract}

KEY WORDS Intermittent Process / Hyperbolic Function / Terminal Group / Polystyrene / Fluorescence Depolarization /

It is well known that polymer chains exhibit orientational relaxation behavior obeying the non-exponential law. This behavior suggests the complexity of the polymer chains to be caused by the vast number of motion. During the last twenty years, many theoretical and experimental studies about local dynamic properties of an isolated chain were reported in order to understand the characteristics of chains itself. Orientational relaxation behavior of a segment in the middle of polymer chain had been studied by many authors. ${ }^{1-7}$ However orientational relaxation of a terminal segment on a polymer chain is not sufficiently discussed.

Various orientational auto-correlations of polymer segments are detected by admirable experimental techniques such as dielectric relaxation, ${ }^{8} \mathrm{NMR},{ }^{9} \mathrm{ESR},{ }^{10}$ and fluorescence depolarization ${ }^{1,6,11}$ method. In particular, the time-resolved fluorescence depolarization method is very powerful to estimate local segmental motions of a polymer chain. Because measured fluorescence anisotropy decay curves directly correspond to the orientational relaxation process of chromophores introduced in a system. ${ }^{12-14}$

In two earlier papers, ${ }^{16,17}$ we discussed the orientational relaxation of the back bone of polystyrene having an anthryl group in the center of its chain (PSAPS). Fluorescence anisotropy decay curves of PSAPS chain were successfully reproducible with hyperbolic function $r(t) \sim r_{0}\left(t / \Gamma_{\text {eff }}\right)^{-\beta}$. $^{16}$ It was found that the origin of hyperbolic anisotropy decay curves of PSAPS chain is the intermittent relaxation ${ }^{16}$ of the segment which is made up of a few monomers. That is to say, orientational relaxation of segments rarely occurs, but the velocity of the relaxation is rapid.

We thus aim to study the relaxation of a terminal segment of polystyrene chain. We will begin by estimating the most appropriate orientational relaxation function for terminal segments according to previous paper. ${ }^{15}$ We discuss the relaxation motion of terminal segments compared with that of inner segments of polystyrene chain.

\section{EXPERIMENTAL}

\section{Samples}

The anthryl group was introduced into the chain end of substantially monodispersed polystyrene (PSA) by the direct reaction of living polystyryl anion with 9chloromethylanthracene prepared in advance from 9anthraldehyde. ${ }^{17}$ Polystyryl monoanion was prepared by living polymerization of styrene with buthyllithium in benzene. Chemical structure of PSA is shown in Figure 1. Values of molecular weight and molecular weight distribution of PSA chains measured with a GPC (Toyo Soda: HLC-802UR) are $5.7 \times 10^{4}$ and 1.18 , respectively. The content of anthryl group determined by UV absorption spectra (Shimadzu: MPS-8000) relative to its theoretical content was $90 \%$.

The sample solution was deaerated by several freezepump-thaw cycles on a high vacuum system. Solvent used in this study composed of tripropionin (glyceryl tripropionate) 0.94 and ethyl acetate 0.06 . The concentration of PSA was kept less than $0.1 \mathrm{wt} \%$ at the final step of preparations.

\section{Measurement}

The sealed Pyrex cylindrical cell was set in the thermostat, and absorption, excitation, emission, and depolarization spectra corrected by a concentrated solution of rhodamine $\mathrm{B}$, and time-resolved emission decay curves of PSA were measured by the PLASMA

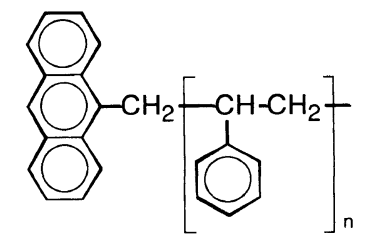

Figure 1. Chemical structure of PSA chain. 
system. ${ }^{15,18}$ The excitation and emission wavelengths were set to 384 and $430 \mathrm{~nm}$, respectively. Measurements were carried out at $25.0,35.0,40.0$, and $45.0^{\circ} \mathrm{C}$.

Fluorescence anisotropy decay curves $r_{\text {obs }}(t)$ were obtained by

$$
r_{\mathrm{obs}}(t) \equiv \frac{I_{\mathrm{VV}}(t)-G I_{\mathrm{VH}}(t)}{I_{\mathrm{VV}}(t)+2 G I_{\mathrm{VH}}(t)}
$$

where $G$ is the compensating factor, which is defined by $G=\int I_{\mathrm{HV}}(t) / \int I_{\mathrm{HH}}(t)$, for anisotropic sensitivity of optics in the used apparatus (time-resolved fluorophotometer, Horiba: NAES-1100). The denominator of above equation corresponds to the fluorescence decay as a function of time $F_{\mathrm{obs}}(t) . I_{\mathrm{VV}}(t), I_{\mathrm{VH}}(t), I_{\mathrm{HH}}(t)$, and $I_{\mathrm{HV}}(t)$ denote polarized fluorescence intensity measured through a band path filter (Toshiba: KL43). Subscripts $V$ and $H$ represent the parallel and the perpendicular direction to the vertical line. The first suffix indicates the direction of an incident light and the second to an emitted light.

\section{Calculation}

Details of calculation were described in previous papers. ${ }^{15,16}$ Deconvolution and curve fitting calculations were carried out on the basis of the quasi-Marquardt algorithm ${ }^{19}$ and the Wahl's method. ${ }^{20}$ The goodness of a fitting calculation was evaluated by the value of the residual sum of squares $\chi^{2}$. Adequate values of variable parameters of a trial function were computed until the variation of $\chi^{2}$ become less than $1 \times 10^{-6}$.

\section{RESULTS AND DISCUSSION}

\section{Fluorescence Anisotropy Decay Curves and Orientational Relaxation Function}

A fluorescence anisotropy decay curve measured at $25^{\circ} \mathrm{C}$ and a calculated anisotropy curve by use of hyperbolic function are shown in Figure 2. If a chromophore attached at the end of polymer chain is able to rotate like a spherical rotator, a measured fluorescence anisotropy decay curve will be a single exponential function. A semi-log plot of the decay curve thus will be on a straight line. However measured data suggest that a probe molecule incorporated at the end of a polymer chain can not rotate isotropically but move in the wake of orientational relaxation motion of a polymer chain. Thus it is important to estimate the most appropriate trial function in order to analyze anisotropy decays, and to understand the orientational relaxation at terminal of a polymer chain.

Functions used in this study are listed in Table I. Values of $\chi^{2}$ indicating the quantitative goodness of a function to measured data are shown in Table II. 'Single' function $r(t) \sim \exp (-t / \tau)$ is not in agreement with data because the value of $\chi^{2}$ is larger than unity. TET function whose function form to be

$$
r(t) \sim 0.265 \exp \left(-\frac{\gamma \sqrt{t}}{4}\right)+0.032 \exp (-\gamma \sqrt{t})+0.103
$$

also does not fit, where $\gamma$ is the torsional rigidity of a chain. TET function is obtained by approximation that angle, which is defined in a Barkley's function, ${ }^{21}$ be-
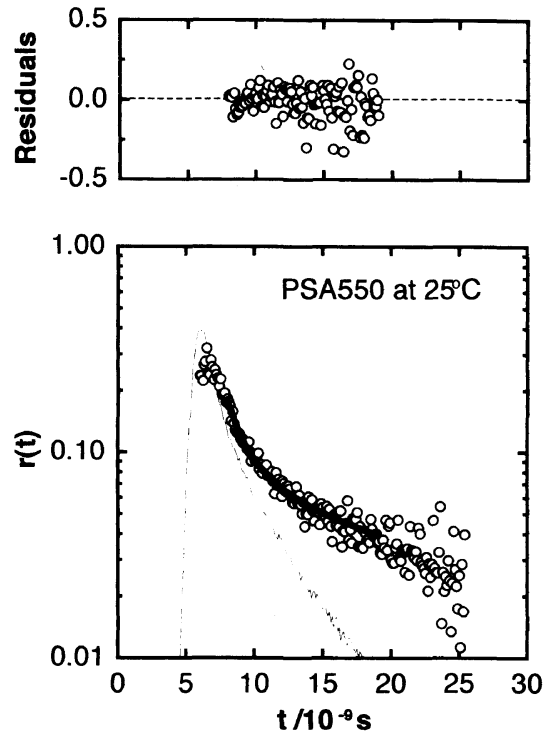

Figure 2. Fluorescence anisotropy decay curve of an anthryl group incorporated in PSA550 chain measured at $25^{\circ} \mathrm{C}$. Thick line is a calculated curve with a hyperbolic function, thin line is a response function of the apparatus, and circles are measured anisotropy decay, respectively. Calculated curve was obtained by the non-linear least square method. Fitting was successfully carried out in the fitting range, because residuals are symmetrically dispersed around zero line. Time scale of this measurement was $25 \mathrm{~ns}$.

Table I. Orientational auto-correlation functions used in this study

\begin{tabular}{ll}
\hline Model & Function \\
\hline Single & $\exp (-t / \tau)$ \\
Double & $a_{1} \exp \left(-t / \tau_{1}\right)+a_{2} \exp \left(-t / \tau_{2}\right)$ \\
JS & $0.578 \exp \left(-t / \tau_{1}\right)+0.422 \exp \left(-t / \tau_{2}\right)$ \\
VJGM & $\exp \left(-t / \tau_{2}\right) \exp \left(t / \tau_{1}\right) \operatorname{erfc}\left(\sqrt{t / \tau_{1}}\right)$ \\
HH & $\exp \left(-t / \tau_{2}\right) \exp \left(-t / \tau_{1}\right) I_{0}(t / \tau)$ \\
GDL & $\exp \left(-t / \tau_{2}\right) \exp \left(-t / \tau_{1}\right)\left(I_{0}\left(t / \tau_{1}\right)+I_{1}\left(t / \tau_{1}\right)\right)$ \\
WH & $(1-a) \exp \left(-t / \tau_{2}\right) \exp \left(-t / \tau_{1}\right) I_{0}\left(t / \tau_{1}\right)+a$ \\
WC & $(1-\alpha) \exp (-t / \tau)+a$ \\
BY & $1 / 2 \sqrt{\pi}\left(\operatorname{erfc}\left(\sqrt{t / \tau_{1}}\right)-\operatorname{erfc}\left(\sqrt{t / \tau_{2}}\right)\right) /\left(\sqrt{t / \tau_{2}}-\sqrt{t / \tau_{1}}\right)$ \\
KWW & $\exp \left(-(t / \tau)^{\beta}\right)$ \\
Hyperbolic & $(t / \tau)-\beta$ \\
TET & $0.265 \exp (-\gamma \sqrt{t} / 4)+0.032 \exp (-\gamma \sqrt{t})+0.103$ \\
Wöessner & $\left(A_{1}+A_{2} \exp \left(-t / \tau_{1}\right)+A_{3} \exp \left(-t / \tau_{1}\right)\right) \exp \left(-t / \tau_{2}\right)$. \\
& $\left(0.578 \exp \left(-t / \tau_{3}\right)+0.422 \exp \left(-t / \tau_{4}\right)\right)$, \\
& {$\left[A_{1}=\frac{1}{4}\left(3 \cos ^{2} \theta-1\right)^{2}, \quad A_{2}=\frac{3}{4} \sin ^{2} 2 \theta, \quad A_{3}=\frac{3}{4} \sin ^{4} \theta\right]$} \\
&
\end{tabular}

Table II. Comparison of $\chi^{2}$ values

\begin{tabular}{cccccc}
\hline Function & $\chi^{2}$ & Function & $\chi^{2}$ & Function & $\chi^{2}$ \\
\hline Single & 3.43 & Double & 1.19 & TET & 10.2 \\
JS & 1.41 & WH & 1.15 & WC & 1.40 \\
GDL & 1.12 & HH & 1.18 & VJGM & 1.40 \\
BY & 1.13 & HYR & 1.17 & KWW & 1.22 \\
\hline
\end{tabular}

tween the transition moment of a chromophore and the axis of a chain is about 35 degree. The torsional model assumed in Barkley's theory for a DNA chain is not good for polystyrene chain.

A detail model for a side group on a polymer chain was proposed by Wöessner ${ }^{22}$ as

$$
r(t) \sim\left(A_{1}+A_{2} \exp \left(-\frac{t}{\tau_{1}}\right)+A_{3} \exp \left(-\frac{t}{\tau_{1}}\right)\right)
$$


$\times \exp \left(-\frac{t}{\tau_{2}}\right) \times\left(0.578 \exp \left(-\frac{t}{\tau_{3}}\right)+0.422 \exp \left(-\frac{t}{\tau_{4}}\right)\right)$

$A_{1}=\frac{1}{4}\left(3 \cos ^{2} \theta-1\right)^{2}, \quad A_{2}=\frac{3}{4} \sin ^{2} 2 \theta, \quad A_{3}=\frac{3}{4} \sin ^{4} \theta$

where $\theta$ is an angle between transition moment of a chromophore and axis of rotation of a chromophore attached to a polymer chain. $\tau_{1}, \tau_{2}, \tau_{3}$, and $\tau_{4}$ are the rotational relaxation time of a polymer side chain, that of main chain, the relaxation times of 3-bond, and lattice motions of a polymer main chain, respectively. It seems useful to apply this function to analyze anisotropy decay curves recorded about terminal segments of polymer chains. However it is difficult to adopt Wöessner's function because it is impossible to determine each parameter independently and accurately with resolution of our apparatus.

As is shown in Table II, values of $\chi^{2}$ obtained about $\mathrm{JS}^{2}{ }^{2} \mathrm{WC}^{23} \mathrm{VJGM}^{24}$ and $\mathrm{KWW}^{25,26}$ functions are larger than $\mathrm{WH},{ }^{27} \mathrm{GDL},{ }^{5} \mathrm{HH},{ }^{4} \mathrm{BY},{ }^{3}$ and hyperbolic (HYP) functions. The variable parameters $\tau_{1}$ and $\tau_{2}$ of $\mathrm{WH}, \mathrm{GDL}$, and $\mathrm{HH}$ functions indicate the relaxation times of the local and global motions on a polymer chain. The base-line parameter $a$ of WH function implies the limit angle in which the local segment wobble. That is, WH, GDL, and HH functions can only express two typical motions among the vast number of motions on a polymer chain. Thus WH, GDL, and $\mathrm{HH}$ functions are not suitable.

BY and hyperbolic functions are able to take into account a number of motion on a polymer chain. Two valuable parameters in BY function $\tau_{1}$ and $\tau_{2}$, respectively, refer to fast and slow cutoff frequencies of motions on a polymer chain. However it is difficult to assign these parameters to molecular motions from Bendler's theory, so that BY function is also not pertinent to a relaxation function. On the other hand, $\beta$ and $\tau$ in hyperbolic function is clearly related to the relaxation motion of a polymer segment as mentioned in the next section. Furthermore $\beta$ is correlated with the distribution function $\Psi(t)$ of the interval time $t$ between relaxations as $\Psi(t)=t^{-1-\beta}$. Hence hyperbolic function is the most favorable among many orientational auto-correlation functions.

In our previous paper, ${ }^{16,28}$ we proposed the ' $\chi^{2}$-map method' in order to clarify the uniqueness of the best fit values of variable parameters contained in a function. The suitability of a function to measured anisotropy decay curves have been estimated by the $\chi^{2}$-map method. Similar maps to reported ones ${ }^{15}$ were obtained for all functions. GDL and $\mathrm{HH}$ functions give a broad wall (Figure 3), so that $\tau_{1}$ is not determined uniquely. For WC function a broad and wall-like peak is found. VJGM, KWW, and hyperbolic functions have a single peak. In particular, the peak shown in $\chi^{2}$-map of a hyperbolic function (Figure 4 ) is more sharp and a little higher than that of VJGM and KWW functions. Thus hyperbolic function is most appropriate to analyze fluorescence anisotropy decay curves.

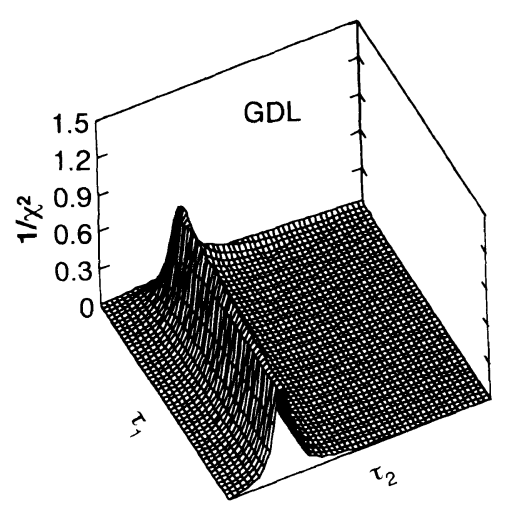

Figure 3. A $\chi^{2}$-map of GDL function. $X, Y$, and $Z$ axes are the value of $\tau_{1}, \tau_{2}$, and $1 / \chi^{2}$, respectively. A broad wall found in this figure represents that one parameter is not determined uniquely.

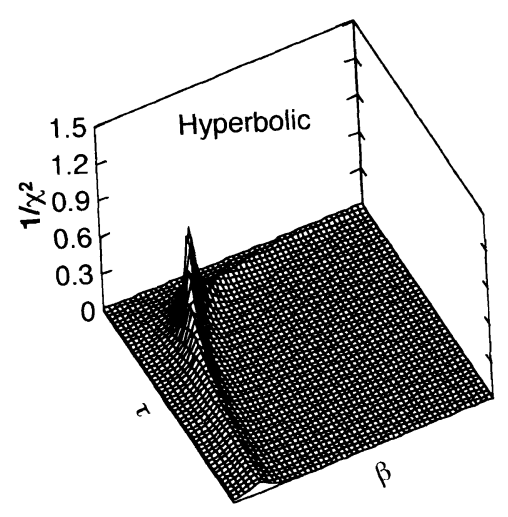

Figure 4. A $\chi^{2}$-map of a hyperbolic function. $X, Y$, and $Z$ axes are the value of $\tau, \beta$ and $1 / \chi^{2}$, respectively. A single and sharp peak is found. This behavior is similar to that about PSAPS chains.

\section{Characteristic Activation Energy of Segments and Mini- mum Reaction Radii}

In our previous paper, ${ }^{16}$ it was found that the origin of hyperbolic fluorescence anisotropy decay curves of PSAPS chain is the intermittent process of orientational relaxation of polymer segments composed with a few monomers. The relaxation of segments was regarded as the reaction between segments and solvent molecules. It was assumed that the state density of segments and the interval time of relaxation following the exponential distribution function. Thus the intermittent relaxation refers to that the relaxation of segments rarely occurs, but the velocity of the relaxation is rapid.

The form of hyperbolic function is

$$
r(t)=r_{0}\left(\frac{t}{\tau_{\text {eff }}}\right)^{-\beta}
$$

where $r_{0}, \tau_{\text {eff }}$ and $\beta$ are a prefactor, the effective relaxation time and a hyperbolic exponent, respectively.

The hyperbolic exponent $\beta$ is correlated to the reference temperature $T_{\mathrm{r}}$, which indicates the distribution of the state density of polymer segments, as

$$
T_{\mathrm{r}}=\frac{T}{\beta+1}
$$

where $T$ is absolute temperature. The values of reference temperature $T_{\mathrm{r}}$ for PSA agrees with that of PSAPS chains $^{16}$ as shown in Table III. Reference temperature 
Table III. Comparison of reference temperatures $T_{\mathrm{r}}$

\begin{tabular}{ccccc}
\hline Sample & PSA550 & PSAPS3200 & PSAPS2500 & PSAPS730 \\
\hline$T_{\mathrm{r}} / \mathrm{K}$ & $189 \pm 4$ & $205 \pm 7$ & $201 \pm 8$ & $202 \pm 7$ \\
\hline
\end{tabular}

Table IV. Characteristic activation energy of polystyrene chain end

\begin{tabular}{lclcr}
\hline Author & Technique & Solvent & $\Delta E / \mathrm{kJ}^{2} \mathrm{~mol}^{-1}$ & Ref. \\
\hline Yang & ELDOR $^{\mathrm{a}}$ & THF & 5.1 & 31 \\
Valeur & FD $^{\mathrm{b}}$ & Tripropionin & 4.2 & 1 \\
Friedrich & ESR & Toluene & 6.1 & 10 \\
& ESR & Toluene & $9.3^{\mathrm{c}}$ & 10 \\
Our result & FD & Tripropionin & 6.6 &
\end{tabular}

${ }^{a}$ Electron-spin double resonance. ${ }^{b}$ Fluorescence depolarization. ${ }^{\mathrm{c}}$ For side-chain. ${ }^{\mathrm{d}} \mathrm{THF}$, tetrahydrofuran.

$T_{\mathrm{r}}$ is related to characteristic activation energy for orientational relaxation of a segment. Characteristic activation energy $E_{\mathrm{a}}$ of polymer segments is given by

$$
E_{\mathrm{a}}=k_{\mathrm{B}} T_{\mathrm{r}}=k_{\mathrm{B}} \frac{T}{\beta+1}
$$

where $k_{\mathrm{B}}$ is the Boltzman constant. As is shown in Table IV, our result of $E_{\mathrm{a}}=6.6 \mathrm{~kJ} \mathrm{~mol}^{-1}$ is not so different from that for reported values. Since the apparent activation energy for orientational relaxation of polymer segments would reach $4 \sim 10 \mathrm{~kJ} \mathrm{~mol}^{-1}$, the apparent activation energy of polymer chain is independent of position along a chain.

The minimum reaction radii $\xi_{0}$ given by

$$
\xi_{0} \sim \frac{\beta}{r_{0} \tau_{\text {eff }}^{\beta}}
$$

depends on the mobility of both the solvent molecule and the polymer segment. Since the mobility of the polymer segment is not varied with temperature in the nallow range of temperature like this experiment, the value of $\xi_{0}$ ought to be samely dependent on temperature with the reciprocal of solvent viscosity $\eta$. The temperature dependence of $\xi_{0}$ shown in Figure 5 obeys the Arrhenius type equation:

$$
\xi_{0} \sim \exp \left(-E_{\mathrm{s}} / k_{\mathrm{B}} T\right),
$$

where $E_{\mathrm{s}}$ is the apparent activation energy of the solvent. Obviously both $\xi_{0}$ for PSA chain and $\eta^{-1}$ are similarly depend on temperature. The values of $E_{\mathrm{s}}$ evaluated by fluorescence anisotropy decay curves of PSA chain was $8.0 \mathrm{~kJ}$ mol. On the other hand, the value of $E_{\mathrm{s}}$ measured directly by a Ubbelohde viscometer was $6.0 \mathrm{~kJ} \mathrm{~mol}^{-1}$. The former value is in agreement with the latter. Therefore the tempreature dependence of $\xi_{0}$ reflects mainly that of solvent.

\section{Simulation of the Degree of Freedom of Chain Terminal \\ As is shown in Figure 5, values of $\xi_{0}$ for PSA chain are about twice times larger than that for PSAPS chain in the range of this experiment. We will focus on the difference in the value of $\xi_{0} . \xi_{0}$ is the minimum reaction radii between a polymer segment and a solvent molecule. The value of $\xi_{0}$ thus reflects the mobility of both the segment and the solvent. The mobility is the product of}

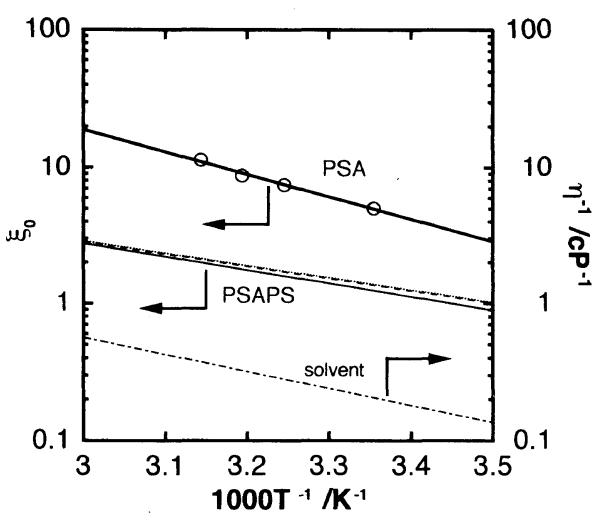

Figure 5. $\xi_{0} v s . T^{-1}$ plot for PSA chain (circles). A thin solid line, a dotted line, and a broken line correspond to temperature dependence of rate constants obtained about PSAPS3200, PSAPS2500, and PSAPS730, respectively. Dot-dash line indicates temperature dependence of the reciprocal of solvent viscosity $\eta$.

the degree of freedom of motion and the probability of diffusing of a species. That is, the minimum reaction radii $\xi_{0}$ is given by

$$
\xi_{0} \sim D_{f, A} P_{A} D_{f, B} P_{B}
$$

where $D_{f, A}$ and $D_{f, B}$ are the degree of freedom of polymer segments and solvent molecules, and $P_{A}$ and $P_{B}$ are the probability of moving of segments and solvent molecules. $P_{A}$ and $P_{B}$ are evaluated by characteristic activation energy of polymer segments $E_{\mathrm{a}}$ and apparent activation energy of solvent $E_{\mathrm{s}}$, respectively. The values of $P_{A}$ and $P_{B}$ are constant at a temperature. For this experiment, the degree of freedom of diffusive motion of solvent molecules is also constant, $D_{f, B}=$ const. Therefore the difference in the values of $\xi_{0}$ at some temperature is reduced to the degree of freedom of segmental motion of a polymer chain. In this section we estimate the degree of freedom of a segment $D_{f, A}$ by the computer simulation. ${ }^{29}$

Let us assume that a polystyrene chain is arranged on a tetrahedral lattice shown in Figure 6(a). Polystyrene chain has by nature phenyl group and hydrogen as the side group. The effect of steric hindrance to the mobility of the chain is already included in the characteristic activation energy of a polymer segment, $E_{\mathrm{a}}$. Thus we regard a polystyrene chain as a chain of the back bone.

A polymer chain is generated along the lattice from the origin to a direction (Figure 6(b)) selected by random numbers. For example, a polymer chain shown in Figure 6(a) is identified as 1746251648 . In this generation process, we adopted the value of energy gap between trans and gauche to be $1.7 \mathrm{~kJ} \mathrm{~mol}^{-1}$. A self-avoiding random chain, whose number of segments is $v$, was obtained as shown in Figure 7.

There are many elemental motions of a polymer chain on a tetrahedral lattice. For example, 1-, 2-, 3-, and 4-bond motions are easily considered (Figure 6(c)-(f)). 1 - and 2-bond motions are only available at the end of a chain. A 2-bond motion requires large free volume around the end of a chain, so that it is difficult to effect such a motion. A 3-bond motion changes the local conformation of a chain as gauche $+\left(g^{+}\right) \rightleftharpoons$ gauche$\left(g^{-}\right)$. Since the local sequence $1-7-3$ is transferred to $3-7-1$, two orientations of segments are modified. The 


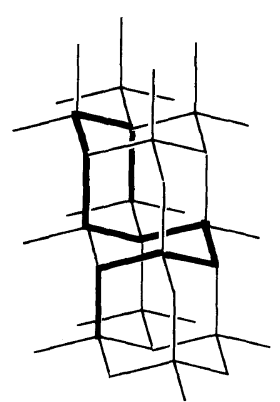

(a)

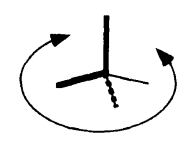

(c)

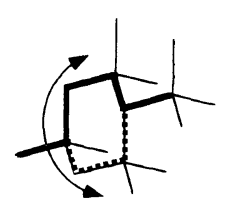

(e)
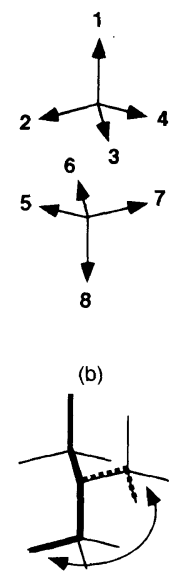

(d)

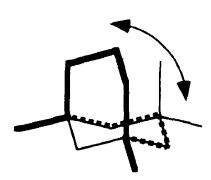

(f)
Figure 6. (a) Tetrahedral lattice and a polymer chain on the lattice. The chain is identified as 1746251648 . (b) Bond vectors along the tetrahedral lattice. (c) 1-Bond motion. (d) 2-Bond motion. (e) 3-Bond motion. (f) 4-Bond motion.

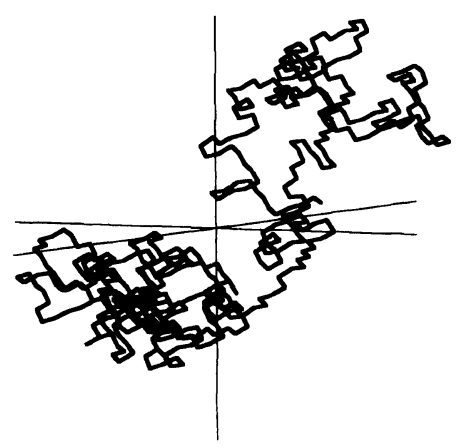

Figure 7. A polymer chain generated on a tetrahedral lattice with energy gap between trans and gauche, $E_{\mathrm{a}}=1.7 \mathrm{~kJ} \mathrm{~mol}^{-1}$. Degree of polymerization in this case is 1400 .

transition from a $g^{+} g^{-}$conformation to a $g^{-} g^{+}$conformation is realized by a 4-bond motion. A 4-bond motion is also not effective as well as a 2-bond motion because the motion creates the excessive contortion on a chain. In this paper, we will limit to consider 1- and 3-bond motions among elemental motions of orientational relaxation of a polymer chain.

Transition probability $P_{\mathrm{tr}}$ for a $j$-bond motion is given by

$$
P_{\mathrm{tr}} \equiv\left(\exp \left(-\frac{E_{\mathrm{a}}}{k_{\mathrm{B}} T}\right)\right)^{j},
$$

where $j$ indicate the number of bonds rotating in the transition process. For 1- and 3-bond motions, values of $j$ are 1 and 3, respectively. When a random number is smaller than $P_{\mathrm{tr}}$ and the moved segment does not overlap with a chain itself, the local conformational change at the position $N$ on a chain is allowed. This operation is carried out from $N=1$ to $N=v$.

The degree of freedom of a segment on a chain is

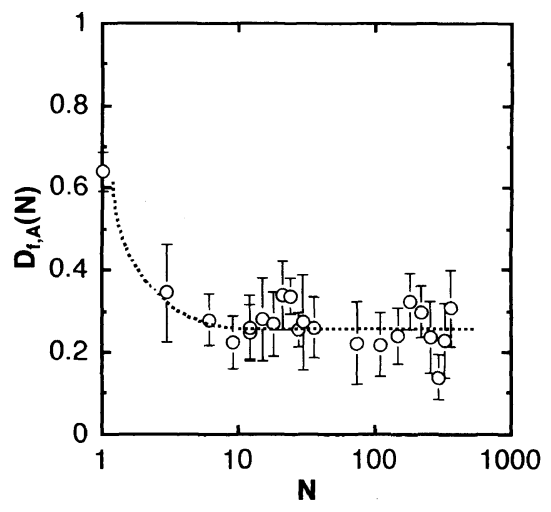

Figure 8. Position dependence of degree of freedom $D_{f, A}(N), N$ is a bond number on a chain. The degree of freedom at the end of a chain is about twice times larger than at the center.

defined as

$$
D_{f, A} \equiv \frac{n}{m}
$$

where $n$ and $m$ are the number of variation and trial, respectively. If a segment is able to move freely, $D_{f, A}=1$ will be obtained, so that the value of $D_{f, A}$ indicates the relative mobility of a segment.

Twenty polymers, whose number of segments is $v=1400$, were generated independently, and the values of $D_{f, A}(N)$ on the position $N$ about each chain were monitored during five thousands operations at temperatures $T=25,30,35,40$, and $45^{\circ} \mathrm{C}$. In the range of temperature, profiles of $D_{f, A}(N)$ were almost same. Figure 8 shows the position dependence of $D_{f, A}(N)$ from the terminal $N=1$ to the center $N=v / 2$ averaged over the range of temperature. The degree of freedom of the terminal segment is about twice times larger than that of "inner" segments.

Gény et al. ${ }^{30}$ simulated the diffusivity of atoms of the chain skeleton. They found the mean-square displacement at the end of a chain to be a few times larger than inner atoms for polyethyleneoxide, poly(methylene oxide), and poly(1,3 dioxolane), and to be about four times larger for poly(tetrahydrofurane), polyoxetane, and polyethylene. Our result about the mobility of a segment at the end of a chain is in good agreement with Gény's study.

Therefore the difference in values of $\xi_{0}$ for a segment at the end and the center of a chain is dominated by the degree of freedom of segments.

\section{CONCLUSION}

The orientational relaxation of terminal segments of polystyrene is successfully reproducible by hyperbolic function, as well as inner segments. Therefore polymer chain dynamics in the time range of nanosecond is explained by the intermittent process of polymer segments.

The temperature dependence of orientational relaxation process is independent of position of a chain, because characteristic activation energies of orientational relaxation at the end and middle of a chain were almost same.

The minimum reaction radii $\xi_{0}$ corresponds not only the apparent activation energy of solvent molecules but 
the degree of freedom of segmental motion on a polymer chain.

\section{REFERENCES}

1. B. Valeur and L. Monnerie, J. Polym. Sci., Polym. Phys. Ed., 14, 11 (1976).

2. A. A. Jones and W. H. Stockmayer, J. Polym. Sci., 15, 847 (1977).

3. J. T. Bendler and R. Yaris, Macromolecules, 11, 650 (1978).

4. C. K. Hall and E. Helfand, J. Chem. Phys., 77, 3275 (1982).

5. J. L. Viovy, L. Monnerie, and J. C. Brochon, Macromolecules, 16, 1845 (1983).

6. D. A. Waldow, M. D. Ediger, Y. Yamaguchi, Y. Matsushita, and I. Noda, Macromolecules, 24, 3147 (1991).

7. K. Ono, Y. Okada, S. Yokotsuka, S. Ito, and M. Yamamoto, Polym. J., 26, 199 (1994).

8. S. Mashimo, Macromolecules, 9, 91 (1976).

9. W. Gronski, T. Schäfer, and R. Peter, Polym. Bull., 1, 319 (1979).

10. C. Friedrich, F. Lauprêtre, C. Noël, and L. Monnerie, Macromolecules, 14, 1119 (1981).

11. T. Sasaki, M. Yamamoto, and Y. Nishijima, Macromolecules, 21, 610 (1988).

12. E. V. Anufrieva and Y. Y. Gotlib, Adv. Polym Sci., 40, 1 (1981).

13. H. Ushiki and K. Horie, "Influence of Molecular Structure on Polymer Photophysical and Photochemical Properties," Vol. 4, N. P. Cheremisinoff, Ed., Marcel Dekker, New York, 1989, p 1.

14. M. D. Ediger, Ann. Rev. Phys. Chem., 42, 225 (1991).
15. F. Tsunomori and H. Ushiki, Polym. J., 28, 581 (1996).

16. F. Tsunomori, H. Ushiki, and K. Horie, Polym. J., 28, 575 (1996).

17. H. Ushiki, K. Horie, A. Okamoto, and I. Mita, Polym. J., 11, 691 (1979)

18. H. Ushiki, Rep. Prog. Polym. Phys. Jpn., 35, 419 (1992).

19. T. Nakagawa and Y. Oyanagi, "Saishou Nijyou-hou Niyoru Jikken-Data Kaiseki," Tokyo Daigaku Syuppan-kai, Tokyo, 1982.

20. P. Wahl, Biophys. Chem., 10, 91 (1979).

21. M. D. Barkley and B. H. Zimm, J. Chem. Phys., 70, 2991 (1979).

22. D. E. Wöessner, J. Chem. Phys., 36, 1 (1962).

23. K. Kinoshita, S. Kawato and A. Ikegami, Biophys. J., 20, 289 (1977).

24. B. Valeur, L. Monnerie, and J. P. Jarry, J. Polym. Sci., Polym. Phys. Ed., 13, 675 (1975).

25. R. Kohlraush, "Annalen der Physik und Chemie," Poggendorf, 1854.

26. G. Williams and D. C. Watts, Trans. Faraday Soc., 66, 80 (1971)

27. T. A. Weber and E. Helfand, J. Phys. Chem., 87, 2881 (1983).

28. H. Ushiki and F. Tsunomori, Rep. Prog. Polym. Phys. Jpn., 36, 373 (1993).

29. T. Yabe, S. Kawata, and M. Fukuta, "Simulation Butsuri," Asakura, Tokyo, 1989.

30. G. Gény and L. Monnerie, J. Polym. Sci., Polym. Phys. Ed., 17, 147 (1979).

31. H. Yang and J. C. W. Chien, Macromolecules, 11, 759 (1978). 